\title{
THE PERIODOGRAM ENVELOPE IN PARAMETRIC AND NON-PARAMETRIC SPECTRAL ESTIMATION
}

Miguel A. Lagunas, Mateo Amengual,Maria E. Forcada

Signal Theory and Communications

E.T.S.I. Telecomunicación, Apdo. 30002 08080 BARCELONA-SPAIN

\section{ABSTRACT}

This work reports the role of periodogram envelope as a non-parametric approach for spectral estimation and its interest in parametric spectral estimation using variational approaches.

The spectral envelope, or its prior estimate the periodogram envelope, represents a very good candidate to start any approach for spectral estimation due to its smooth character, its robustness againts flat noise spectra and its statistical stability.

Setting envelope constraints in variational spectral estimation the resulting estimate gains in statistical stability yet preserving the high resolution of the best reported methods.

Together with the non-parametric approaches, the case of line spectra is briefly described to evidence the potential of envelope associated matrix in line spectrum. This allows the designer to convert SUD and MUSIC methods to the new starting function.

\section{INTRODUCTION}

It is assumed that given a signal $x(n)$, its envelope and its instantaneous frequency provides a better description of the involved physical phenomena.

This work deals with the role of the spectral envelope $E_{p}(\omega)$ defined as the envelope of the power spectral density $S_{p}(\omega)$ of a given random process $\{x\}$. To be more specific it will be the square of the envelope $\mathrm{E}_{\mathrm{p}}{ }^{2}(\omega)$ the function under study; and, the closeness it can provide to the accurate estimation of the actual power spectral density. The point of special interest around will be up to what degree the periodogram envelope $E_{p}{ }^{2}$, defined as the envelope of the magnitud of DFT $[x(n)]$, could be used as a starting point to obtain the distribution of the signal power in the frequency domain. The reader will notice that due to the non-linear character of the spectral estimation problem any candidate added to the basic a priori information (i.e. the periodogram) will have a considerable potential to become usefull to our final goal which is the accurate estimation of the power spectral density.

The periodogram envelope is a fourth order function and, in this sense this work can be encompassed as how to use high order moments of the signal in spectral estimation. However, the concept of envelope results by itself self explanatory in many senses, and it seems to be better to concentrate efforts in this special fourth order function

This work is supported by a PLANICYT grant. without a general backgroung or framework for other nonlinear functions.

\section{THE SPECTRAL ENVELOPE}

Given the signal periodogram $P(\omega)$, we define the spectral envelope as the magnitude of the function $A_{P}(\omega)$, named as analytical periodogram because its inverse Fourier transform is a causal function.

$$
\begin{aligned}
& A_{P}(\omega)=P(\omega)+j H_{P}(\omega) \\
& F^{1}\left[A_{P}(\omega)\right]=0 ; \quad n<0
\end{aligned}
$$

The inverse Fourier transform is as denoled in (2).

$$
F^{-1}\left[A_{P}(\omega)\right]=\begin{array}{ll}
0 & ; n<0 \\
r(0) / 2 & ; n=0 \\
r(n) & ; n>0
\end{array}
$$

Note that the analytic periodogram is in correspondence with the analytic signal and its inverse Fourier transiorm obeys to the concept of causal autocorrelation function introduced some years ago by J. Cadzow.

Working over, or estimating, the analytic spectrum $A_{s}(\omega)$ represents somehow the same problem; the spectral estimate $S(\omega)$ can be derived from the analytic estimate just taiking its real part.

$$
S(\omega)=R_{e}\left[A_{S}(\omega)\right]
$$

The difference resides in the powerfull mathematica framework behind analytic functions which appears in the analytic periodogram and not in the original function.

Once again the procedure of J.P. Burg should be mentioned in one of the most unknown aspect it has. Maximum entropy or AR modelling of data time series was reported in order to smooth the data periodogram; in fact this was the main goal when, with a few paramelers, the authors (Parzen, Burg, etc.) represented a data record power distribution. The high performance of MEM in short data records was an additional result, but as concerns resolution for long data records MEM is not in competition with the periodogram.

It is easy to show that the periodogram envelope is smoother than the periodogram. Using the magnitude/phase representation of the analytic spectrum $\left(E_{p} / \phi_{p}\right)$ and (1) we can arrive to (4).

$P(\omega)=E_{p}(\omega) \cdot \cos \phi_{p}(\omega)$

Note in (4) that the periodogram has the variations of

CH2673-2/89/0000-2174 \$1.00 (C) 1989 IEEE 
the phase which introduces tru peaky character in $P(\omega)$ when compared with the periodogram envelope.

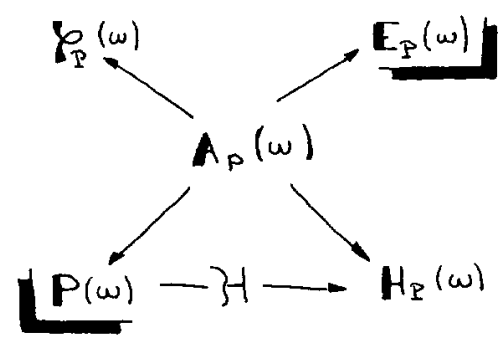

Also it is interesting to check that $\varphi(\omega)$ is the minimum phase associated to the magnitude (i.e. the spectral envelope $\left.E_{p}(\omega)\right)$. This reveals that given $E_{p}(\omega)$ we can obtain $P(\omega)$ and viceversa. To prove this last sentence, note that the real part of $A_{P}(s)$ is always positive and, as a consequence, its inverse shares the same property. Because $A_{P}(s)$ and $A_{p}{ }^{-1}$ (s) have positive real parts we can conclude that both ceros and poles are inside the unit circle and, in conclussion, $A_{p}(s)$ is a minimum phase function.

In summary, at the expense of further comments, the spectral envelope does not represents any loss of spectral information. The only differences to be expected between using the periodogram or its envelope as starting information in a spectral estimation procedure is the nonlinear character involved in such a procedures.

\section{NON-PARAMETRIC SPECTRAL ENVELOPE ESTIMATION}

The periodogram envelope can be derived from the analytic spectrum taking into account that its Fourier inverse is just the causal autocorrelation function,

$$
\begin{aligned}
& A_{P}(\omega)=S^{\top} \quad \begin{array}{ccccc}
r_{0} / 2 & r_{1} & \ldots & r_{Q} \\
0 & 0 & r_{0} / 2 & \ldots & r_{Q-1}
\end{array} \\
& \ldots \ldots \ldots \ldots \ldots \\
& 0 \begin{array}{cccc}
0 & \cdots & r_{0} / 2
\end{array} \\
& =\underline{S}^{\top} \mathrm{R}_{C} \mathrm{~S}
\end{aligned}
$$

where $\mathbf{S}$ is the steering vector defined as $S_{i}=\exp (j i \omega)$ and $r_{0}, r_{1}, \ldots, r_{Q}$ are the first $Q$ estimated autocorrelacion samples.

The spectral envelope is derived laking the magnitude of the analytic spectrum.

The main advantage, and this is the difference with the periodogram, is that the spectral envelope is always positive and does not depend on the data or lag window used to estimate $r_{Q}$ in this respect.

In Figure 1 the reader can see that, in the problem of line spectra, the periodogram envelope provides an smoother behavior than the periodogram and, what is going to be of capilal importance in next section, more statistical stability than the periodogram.

Other formulation of interest to derive the periodogram envelope arises from considering the expressions of the periodogram and its Hilbert transform as quadratic forms. $+d B$.

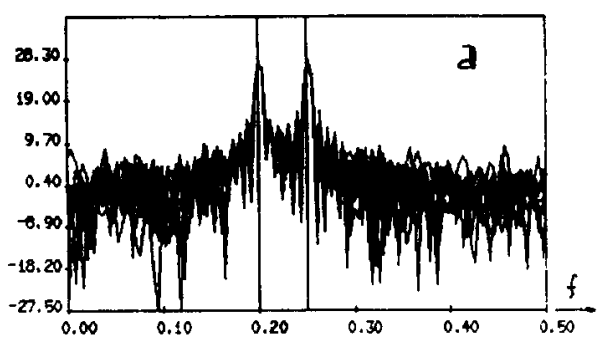

$+d B$.

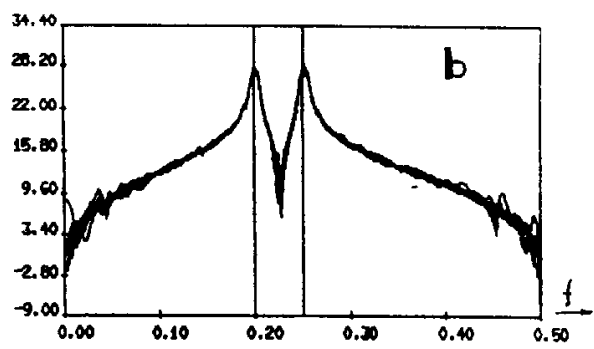

Figure 1. (a) Ten periodograms and in (b) the corresponding envelopes. Data records of 128 samples with two sinusoids at normalized frequencies of 0.2 and 0.25 in white noise at SNR equal to $10 \mathrm{~dB}$.

$$
\begin{aligned}
& P(\omega)=S^{\top} \cdot B \cdot S \\
& j H_{P}(\omega)=S^{\top} \cdot B_{a} \cdot S
\end{aligned}
$$

where $B+B_{a}$ is the upper triangular matrix of (5). Thus, from (6) we can derive the magnitude of the analytic spectrum as it is shown in (7), where vector $\mathbf{s}$ * inciudes a $-\pi / 2$ phase in every component.

$$
\begin{gathered}
E_{P}^{2}(\omega)=\left(S^{\top} \underline{R S}\right)^{2}+\left(\frac{1}{j} S^{\top} B_{a} S\right)^{2}= \\
=\left(S_{T} R S\right)^{2}+\left(S^{*} B_{a} S\right)^{2}
\end{gathered}
$$

Now, using the ideas reported by Lagunas [5] in the topic of Rayleigh estimates for spectral estimation, a new spectral envelope estimate can be derived from (8).

$$
E_{S}{ }^{2}(\omega)=\underline{S}^{\top} \phi S=\left(S_{T} B^{2} \underline{S}\right)+\left(\underline{S} *{ }^{\top}{ }_{a}{ }^{2} S\right)
$$

Where matrix $\&$ can be easily constructed from $\mathbf{B}$ and $\mathrm{B}_{\mathbf{a}}$ or directly from the causal autocorrelation function as shown below :

- Given $r()=$. causal autocorrelation function Compute the autocorrelation of $r($.$) and denote as$ $\phi$ (.) the corresponding values

Form $t$ in a Toeplitz fashion with the first $Q$ values of the sequence $\phi($.

Both $E_{p}$ and $E_{S}$ can be used as an alternative to the classical periodogram with the following advantages : (a) $E($.$) is always positive; (b) E($.$) has not the peaky or noisy$ 
character of the periodogram; (c) $E($.$) has very good$ statistical stability and results very robust for practical applications.

\section{PARAMETRIC SPECTRAL ENVELOPE ESTIMATION}

From the methods reported currently, the only one which provides a variational framework was due to Lagunas et al. [1] proving that MEM as objective with correlation and cepstrum constraints results in an ARMA model for the process under analysis. The algorithm was solved by Musicus [2] which converted the cepstrum constraints in first order information and after using the ideas of Mullis and Roberts [4] for mixed first and second order information the ARMA parameters were obtained.

ARMA models for the analytic spectrum are derived from the $z$-transform of the causal autocorrelation $r_{a}(q)$.

$$
A_{s}(z)=\frac{r_{0}}{2}+\sum_{q=1}^{\omega} r_{q} \cdot z^{-q}
$$

Naming $N(z)$ and $B(z)$ the cero-pole function of the analytic spectrum the expression for the spectral envelope and the spectral density follows :

$$
\begin{aligned}
& A_{S}(z)=\frac{N(z)}{B(z)} ; S(z)=\frac{N(z)}{B(z)}+\frac{N(1 / z)}{B(1 / z)} \\
& E(z) E(1 / z)=N(z) N(1 / z) / B(z) B(1 / z)
\end{aligned}
$$

Note that envelope constraints are a nice candidate for variational spectral estimation because differs from the cepstrum in many important aspects. The envelope obeys to a rational model with the same poles that the actual spectral estimate. Due to the previous comment there is no bandwidth extension as in cepstrum . In using a small set of constraints the statistical stability of the selected ones will provide this behavior to the associated spectral estimate yet preserving the involved high resolution of ARMA estimates from a variational approach. The envelope or the squared envelope is a fourth order function that, used together with the classic second order a.c.f., represents a steep ahead in spectral estimation techniques.

Using correlation and envelope constraints in variational spectral estimation is done by finding an extremum for $\phi(0)$ constrained with $Q$ lags of a.c.f. and $Q$ lags of function $\phi($.$) excluding the cero lag. Thus, using that$ the envelope is the magnitude of the analytic spectrum the problem could be stated as it is sohwn in (11).

Objective :

$$
\left.\int_{-\pi}^{\pi}\left|A_{S}(\omega)\right|^{2} d \omega\right|_{\text {EXTREMUM }}=\phi^{e}(0)
$$

Correlation constraints (causal a.c.f.)

$$
\frac{1}{2 \pi} \int_{-\pi}^{\pi} A_{S}(\omega) \cdot \exp (j n \omega) d \omega=r^{c}(n) ; n=0, Q
$$

Envelope constraints

$$
\begin{gathered}
\frac{1}{2 \pi} \int_{-\pi}^{\pi}\left|A_{S}(\omega)\right|^{2} \exp (j m \omega) d \omega=\phi(m) \\
m=1, Q
\end{gathered}
$$

Where

$$
\phi(m)=\frac{1}{2 \pi} \int_{-\pi}^{\pi} E_{P}^{2}(\omega) \exp (j m \omega) d \omega
$$

Using the Lagrange multipliers and setting the derivatives with respect $A_{s}($.$) equal to cero an ARMA model$ is obtained of the analytic spectrum [3].

$$
\begin{aligned}
& A_{S}(z)=\frac{N(z)}{B(z)} \\
& N(z)=\Sigma c(j) z^{-j} \\
& B(z)=\Sigma b(j) z^{-j}
\end{aligned}
$$

The algorithm to find vectors of coefficients $\mathrm{s}$ and $\mathrm{b}$ starts with the autocorrelation constraints.

$$
\mathbf{c}=\underline{\mathrm{R}}_{\mathrm{C}} \cdot \mathbf{b}
$$

where $\mathrm{B}_{\mathrm{C}}$ is the upper diagonal matrix of (5).

Then, the envelope constraints evolve to (15), where the asterisc in $\phi($.$) denotes that the cero value is unknown$ because it is the objective.

$$
\frac{N(z)}{B(z)} \frac{N(1 / z)}{B(1 / z)}=\phi^{*}(z)=\sum_{-\infty}^{\infty} \phi(m) z^{-m}(15)
$$

$N(1 / z) / B(1 / z)$ can be traslated to the sample domain using the autocorrelation constraints.

$$
\begin{aligned}
& N(z) \frac{N(1 / z)}{B(1 / z)}=\phi^{*}(z) \cdot B(z) \\
& B_{C}^{\top} \cdot c=m^{*} b
\end{aligned}
$$

Finally, introducing (14) in (16.b) the eigenvalue problem results.

$$
\left(\mathrm{B}_{c}^{\top} \mathrm{R}_{\mathrm{c}}\right) \cdot \mathrm{b}=\phi^{*} \cdot \mathrm{b}
$$

It is worthwhile to note that $\phi^{*}$ includes the value which is the objective $\phi_{e}(0)$. Naming the difference between $\phi(0)$ (obtained from the periodogram envelope) and $\phi^{e}(0)$ as $\Delta$, it is easy to prove that :

$$
\Phi^{*}=0-\Delta \cdot 1
$$

Thus, solving (19) for the minimum eigenvalue will result in the desired solution.

$$
\left(\boldsymbol{c}^{*}-\mathrm{B}_{\mathrm{c}}^{\top} \mathrm{B}_{\mathrm{c}}\right) \mathrm{b}=\lambda \mathrm{b}
$$

see [4] for details in solving (17) or (19).

Figure 2 shows the comparison between ARMA spectral estimation using correlation and cepstrum constraints (a) and correlation and envelope constraints (b). The superiority of the herein reported approach becomes evident. 

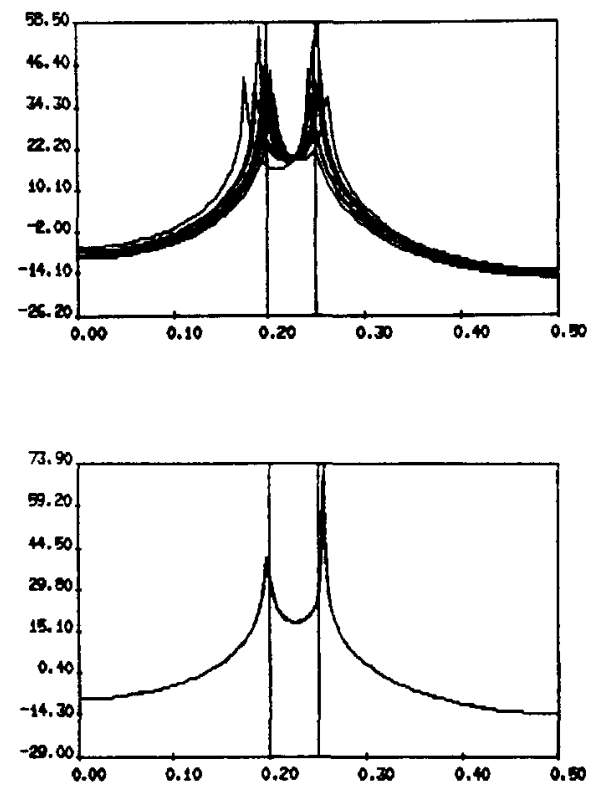

Fig. 2. Cepstrum and correlation constraints (a); and, correlation envelope constraints (b). The signal records are the same as in Figure 1 with 64 samples.

\section{LINE SPECTRA FROM THE ENVELOPE MATRIX}

This section is devoted to complete the role of envelope in spectral estimation, and more concrectly in the problem of line espectra and SVD or MUSIC, by showing the superiority of matrix over the classic matrix $B$ as starting function in these procedures.

As an example of use, in Figure 3 the inverse Fourier transform of the eigenvector associated to the minimum eigenvalue of $R$ and $\Phi$ is represented for 10 records of the same signal that in figure 2. Again, and perhaps more dramatic than before, the superiority of the envelope matrix results clear in front of the periodogram matrix.
$E_{p}^{2}(\omega)$
Inv. F. Transform
$\phi($.
$\Phi$
$P(\omega)$
Inv. F. Transform. $\mathrm{r}($.

(20)
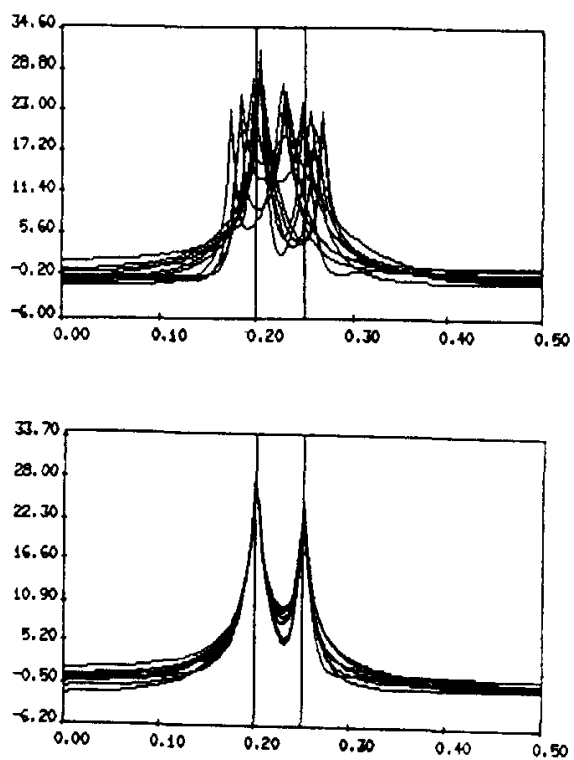

Fig. 3. Inverse of the magnitude of the minimum eigenvalue eigenvector (a) for the periodogram associated matrix $B$ and for the envelope associated matrix $\Phi$ in (b). The signal records (10 records) are the same that in figure 2 .

\section{REFERENCES}

[1] M.A. Lagunas et al. "ARMA Model Maximum Entropy Spectral Estimation" IEEE Trans. on Acoutics, Speech and Signal Processing, ASSP-32, no. 5, pp. 984 990, October 1984

[2] B.R. Musicus and A.M. Kabel "Maximum Entropy PoleZero Estimation" Proc. ICASSP-86. Tokyo, paper 27.12, 1986

[3] M.A. Lagunas "The Variation Approach in Spectral Estimation" Proc. EUSIPCO-86, La Haye, The Netherlands, Sept. 2-5, Ed. North-Holland

[4] C.T. Mullis and R.A. Roberts "The use of Second-order Information in the Approximation of Discrete-Time Linear Systems" IEEE-ASSP, Vol. 24, no.3, June 1976

[5] M.A. Lagunas and M. Cabrera "Rayleigh Spectra Estimates" Submitted for publication at the IEEEASSP Transactions

The reasons for this performance surely are based, without further work, in the statistical stability of the periodogram envelope. As intuitive reasoning it can be said that the Hibert transform contribution to the envelope in (21) support an enhancement of the signal zones at the expense of reducing the flat noise spectra. Also note that squaring a function tends to reduce and provide better separation between the noise and the signal subspace. 